\title{
Usefulness of inflammatory biomarkers in discriminating between bacterial and aseptic meningitis in hospitalized children from a population with low vaccination coverage
}

Ewelina Gowin ${ }^{1}$, Jacek Wysocki², Dirk Avonts³ ${ }^{3}$ Danuta Januszkiewicz-Lewandowska ${ }^{4-6}$, Michal Michalak

\author{
${ }^{1}$ Department of Family Medicine, Poznan University of Medical Sciences, Poznan, \\ Poland \\ 2Department of Health Promotion, Poznan University of Medical Sciences, Poznan, \\ Poland \\ ${ }^{3}$ Family Medicine Department, University of Ghent, Ghent, Belgium \\ ${ }^{4}$ Department of Molecular Pathology, Institute of Human Genetics, Polish Academy \\ of Sciences, Poznan, Poland \\ ${ }^{5}$ Department of Oncology, Hematology and Bone Marrow Transplantation, \\ Poznan University of Medical Sciences, Poznan, Poland \\ ${ }^{6}$ Department of Medical Diagnostic, Poznan, Poland \\ ${ }^{7}$ Department of Biostatistics, Poznan University of Medical Sciences, Poznan, Poland
}

\author{
Corresponding author: \\ Ewelina Gowin MD, PhD \\ Department \\ of Family Medicine \\ Poznan University \\ of Medical Sciences \\ 49 Przybyszewskiego St \\ 60-355 Poznan, Poland \\ Phone: +48618691147 \\ E-mail: \\ ewego@poczta.onet.pl
}

Submitted: 25 May 2014

Accepted: 23 July 2014

Arch Med Sci 2016; 12, 2: 408-414

DOI: 10.5114/aoms.2016.59269

Copyright (c) 2016 Termedia \& Banach

\section{Abstract}

Introduction: Neisseria meningitidis and Streptococcus pneumoniae are the most frequent pathogens responsible for meningitis beyond the neonatal period. Aseptic meningitis is a disabling condition, but bacterial meningitis if left untreated is $100 \%$ fatal. The aim of the study was to analyze the usefulness of biochemical and hematological parameters in distinguishing between bacterial and non-bacterial meningitis in children with meningitis from a population with low rates of vaccination against S. pneumoniae and $N$. meningitidis.

Material and methods: This study is a retrospective chart review of children hospitalized with meningitis. In patients with aseptic and bacterial meningitis the following parameters were compared: C-reactive protein, D-dimers, fibrinogen, glucose level, and leukocyte level, and in cerebrospinal fluid, protein, glucose, and leukocyte concentrations were analyzed. Number of points in the Bacterial Meningitis Score (BMS) was calculated. The predictive value of each parameter to distinguish between bacterial and aseptic meningitis was evaluated.

Results: In total, 129 patients were included in the study: 65 diagnosed with bacterial meningitis and 64 with aseptic meningitis. Bacterial and aseptic meningitis were statistically significantly different based on each analyzed parameter $(p<0.000001)$. Among children with aseptic meningitis $42(66 \%)$ scored 0 points in the BMS, while all the children with bacterial meningitis had at least one point.

Conclusions: In children with meningitis inflammatory biomarkers differ statistically significantly depending on the etiology - bacterial or aseptic. Serum concentration of C-reactive protein higher than $80 \mathrm{mg} / \mathrm{dl}$ is a useful marker of bacterial etiology of meningitis. A high Bacterial Meningitis Score is indicative for bacterial meningitis.

Key words: bacterial meningitis, aseptic meningitis, C-reactive protein, neuroinfection. 


\section{Introduction}

Meningitis is an acute inflammation of tissues surrounding the brain. The inflammatory process is induced by locally produced cytokines such as interleukin (IL)-1, IL-6, and tumor necrosis factor (TNF)- $\alpha$ in response to various pathogens [1]. Infectious meningitis is caused by entry of pathogens into the sterile cerebrospinal fluid. The main invasion route is through the blood, carrying pathogens from colonized mucous membranes in distant sites of infection with sinusitis, pneumonia or otitis media [2,3]. Fever, headache, changes in mental status and meningismus are the typical signs of meningitis. Neisseria meningitidis and Streptococcus pneumoniae are the two most frequent pathogens responsible for meningitis beyond the neonatal period [2-4]. In aseptic meningitis, there are clinical signs of meningitis and inflammatory changes in cerebrospinal fluid along with negative cultures prior to antibiotics administration. Most cases of aseptic meningitis are caused by viruses, but they can also be in duced by drug hypersensitivity, vasculitis or tumor. Aseptic meningitis is a disabling condition, but bacterial meningitis if left untreated is $100 \%$ fatal [1].

Diagnosis of bacterial meningitis is based on a positive culture of cerebrospinal fluid (or detecting bacterial material by polymerase chain reaction - PCR), along with typical clinical symptoms (fever, headache, neck stiffness). The diagnosis is confirmed when cerebrospinal fluid examination reveals signs of inflammation. In bacterial meningitis, cerebrospinal fluid examination may show pleocytosis with neutrophil predominance, low glucose and increased protein concentration [5]. Compared with bacterial meningitis, aseptic meningitis has a lower protein concentration in the cerebrospinal fluid, a normal glucose level and a predominance of lymphocytes [6].

With increasing coverage of vaccination against bacterial meningitis in the vulnerable population, the proportion of aseptic meningitis is increasing [7]. In countries with high percentages of vaccinations against the three main bacterial pathogens, bacterial meningitis is rare. Routine vaccinations against Haemophilus influenzae type $b(\mathrm{Hib})$ reduced the number of $H$. influenzae meningitis cases [8]. A similar phenomenon is observed with S. pneumoniae and N. meningitidis vaccinations $[9,10]$. But in the above-mentioned pathogens, there are several serotypes, so the effectiveness of vaccines is lower, and cases of pneumococcal or meningococcal meningitis are still reported. Before the vaccination era, encapsulated strains of $H$. influenzae were responsible for almost $45 \%$ of cases of bacterial meningitis in children [8]. Among children with symptoms of neuroinfection the majority will have disease of non-bacterial origin. But still there will be a group of patients with bacterial meningitis, in which proper antibiotic treatment must be administered immediately. Clinical symptoms and signs cannot discriminate between bacterial and aseptic meningitis. Analysis of inflammatory biomarkers will offer additional diagnostic information to distinguish between a bacterial or non-bacterial origin.

\section{Material and methods}

This study is a retrospective chart review of all children hospitalized with clinical suspicion of meningitis. They were admitted to the infectious diseases ward of the Children's Hospital between January 2008 and December 2012. The described Infectious Diseases Ward serves for almost the whole children population of the Wielkopolska region: $10 \%$ of the Polish population (almost 600000 children). All children in Poland have mandatory health care insurance. Patients were identified using the international classification of diseases tenth revision (ICD-10). All the analyzed children were previously healthy, with no chronic diseases, and received all compulsory vaccinations. Inclusion criteria were: clinical symptoms at admission suggesting meningitis (fever, neck stiffness, headache) and inflammatory changes in cerebrospinal fluid. Exclusion criteria: severe chronic condition, concomitant varicella. Data abstracted from charts included the age of the child, occurrence of rash, seizures, vomiting and several biochemical and hematological biomarkers. In patients with aseptic and bacterial meningitis the following parameters taken on admission were compared: C-reactive protein (CRP), D-dimers, fibrinogen, glucose level, and leukocyte level, and in cerebrospinal fluid, protein, glucose, and leukocyte concentrations were analyzed. C-reactive protein was measured by immunoturbidimetric assay (Tina-quant CRP detection method; Roche Diagnostics), and procalcitonin levels were measured using the Vidas B.R.A.H.M.S PCT assay (bioMérieux). Number of points in the Bacterial Meningitis Score (BMS) was calculated [11]. The Bacterial Meningitis Score was developed by $\mathrm{Ni}$ grovic et al. [11]. It is used in children with meningitis to indicate those with a low risk of bacterial infection. The following parameters are assessed: positive Gram stain, neutrophil count higher than or equal to $\geq 1000 / \mu \mathrm{l}$ and protein concentration $\geq 80 \mathrm{mg} / \mathrm{dl}$, neutrophil count in peripheral blood $\geq 10000 / \mu \mathrm{l}$, seizure occurrence. Patients with none of these parameters are at low risk of having bacterial meningitis. The predictive value of each parameter to distinguish between bacterial and aseptic meningitis was evaluated. 


\section{Statistical analysis}

Comparison of age and all clinical parameters between bacterial and aseptic meningitis patients was performed by Student's $t$-test when data were normally distributed and by the Mann-Whitney test when data did not follow a normal distribution. Normality was analyzed by the Shapiro-Wilk test. The comparison between normal and abnormal ranges of analyzed parameters and types of meningitis was performed using the $\chi^{2}$ test of independence. For significant parameters the odds ratio and 95\% confidence interval (Cl) were assessed.

Receiver operating characteristics (ROC) curves were calculated to determine the potential of analyzed parameters to discriminate between bacterial and aseptic meningitis patients. An optimal cutoff point was calculated according to the highest accuracy (minimal false negative and false positive results). The area under the ROC curve (AUC) was used to check the prognostic value of a particular parameter. For given cut-off points predicting bacterial meningitis, sensitivity and specificity were calculated. Ethical approval for data management was obtained from the Ethics Committee of the Poznan University of Medical Sciences.

\section{Results}

In total, 129 patients (aged 1 month to 18 years) were included in the study, among whom 65 were diagnosed with bacterial meningitis and 64 with aseptic meningitis. The analyzed group consisted of patients aged 28 days to 18 years old. Mean age was 94 months (7 years 10 months). Mean age of children with bacterial meningitis was 71 months (5 years 11 months) and was significantly lower from the mean age of children with aseptic meningitis ( $p<0.001), 117$ months (9 years 9 months).

Mean values of inflammatory parameters in blood are presented in Table I. The distribution of values is presented in Table II. Bacterial and aseptic meningitis were statistically different based on each analyzed parameter.

In $86 \%$ of the patients with bacterial meningitis, protein concentration was above $0.8 \mathrm{~g} / \mathrm{l}$, compared to $25 \%$ of the children with aseptic meningitis ( $\mathrm{OR}=18.67 ; 95 \% \mathrm{Cl}: 7.56-46.06)$. The leukocyte level was above 1000 per $\mu$ in $92 \%$ of the patients with bacterial meningitis, compared to $63 \%$ of the patients with aseptic meningitis (OR = 6.28; 95\% Cl: 2.20-17.93).

In aseptic meningitis, glucose level was below $50 \mathrm{mg} / \mathrm{dl}$ in $76 \%$ of patients. A glucose level of $>50 \mathrm{mg} / \mathrm{dl}$ was 7 times more frequent in the group with aseptic meningitis, compared with bacterial meningitis ( $\mathrm{OR}=7.33$; 95\% Cl: 3.35-16.04).

Among children with aseptic meningitis, 42 (66\%) scored 0 points in BMS, while all the children with bacterial meningitis had at least one point. Patients with more than 3 points in the BMS were 115 times more likely than patients with fewer BMS points to be diagnosed with bacterial meningitis (OR = 115.04; 95\% Cl: 14.95-885.05). Table III presents the predictive value of selected parameters.

No significant difference was observed in seizure frequency between patients with bacterial and aseptic meningitis (11 of all patients). Hemorrhagic rash was 10 times more common in patients with bacterial infection, but it was observed in $23 \%$ of patients. Headache was present in $69 \%$ of all patients, and vomiting (67\%) was more common in patients with aseptic meningitis (OR was respectively 6.8 and 2.3 ).

Table I. Mean values and SD of selected biochemical and hematological parameters in patients with bacterial and aseptic meningitis

\begin{tabular}{|c|c|c|c|c|c|}
\hline \multirow[t]{2}{*}{ Parameter } & \multicolumn{2}{|c|}{ Bacterial } & \multicolumn{2}{|c|}{ Aseptic } & \multirow[t]{2}{*}{$P$-value } \\
\hline & Mean & SD & Mean & SD & \\
\hline CRP $[\mathrm{mg} / \mathrm{l}]$ & 220.41 & 83 & 6.87 & 12.24 & $<0.000001$ \\
\hline D-dimers $[\mu \mathrm{g} / \mathrm{l}]$ & 2403.15 & 1721.89 & 388.40 & 93.19 & $<0.000001$ \\
\hline Leucocytes $[1000 / \mu l]$ & 18.64 & 8.18 & 10.36 & 6.07 & $<0.000001$ \\
\hline Fibrinogen [g/l] & 7.12 & 1.94 & 3.29 & 1.69 & $<0.000001$ \\
\hline Glucose [mg/dl] & 121.91 & 52.10 & 95.61 & 25.63 & 0.001006 \\
\hline Protein CSF [g/l] & 2.37 & 1.92 & 0.67 & 0.55 & $<0.000001$ \\
\hline Leukocytes CSF $[n / \mu l]$ & 4911.18 & 5614.44 & 439.26 & 694.09 & $<0.000001$ \\
\hline Glucose CSF [mg/dl] & 34 & 24.66 & 56 & 16.44 & $<0.000001$ \\
\hline BMS & 2.97 & 1.06 & 0.42 & 0.66 & $<0.000001$ \\
\hline
\end{tabular}

BMS - Bacterial Meningitis Score, CRP - C-reactive protein, CSF - cerebrospinal fluid, SD - standard deviation. 
Usefulness of inflammatory biomarkers in discriminating between bacterial and aseptic meningitis in hospitalized children from a population with low vaccination coverage

Table II. Distribution of values of selected parameters in patients with bacterial and aseptic meningitis

\begin{tabular}{|c|c|c|c|}
\hline Parameter & $\begin{array}{c}\text { Bacterial } \\
n=65\end{array}$ & $\begin{array}{c}\text { Aseptic } \\
n=64\end{array}$ & $P$-value \\
\hline \multicolumn{4}{|l|}{ CRP $[\mathrm{mg} / \mathrm{l}]:$} \\
\hline Normal & 0 & 49 (76.56\%) & $<0.0001$ \\
\hline Above normal range & $65(100 \%)$ & $15(23.44 \%)$ & \\
\hline \multicolumn{4}{|l|}{$\mathrm{OR}=418.35 ; 95 \% \mathrm{Cl}: 24.4-7167.3$} \\
\hline \multicolumn{4}{|l|}{ D-dimers $[\mu g / l]:$} \\
\hline Normal range & $2(3.08 \%)$ & $27(72.97 \%)$ & $<0.0001$ \\
\hline Above normal range & $63(96.92 \%)$ & $10(27.03 \%)$ & \\
\hline \multicolumn{4}{|l|}{$\mathrm{OR}=85.05 ; 95 \% \mathrm{Cl}: 17.447-414.63$} \\
\hline \multicolumn{4}{|l|}{ Fibrinogen $[\mathrm{g} / \mathrm{l}]$ : } \\
\hline Normal & 0 & $31(67.39 \%)$ & $<0.0001$ \\
\hline Above normal range & 65 (100\%) & $15(32.61 \%)$ & \\
\hline \multicolumn{4}{|l|}{$\mathrm{OR}=266.23 ; 95 \% \mathrm{Cl}: 15.42-4596.7$} \\
\hline \multicolumn{4}{|l|}{ Glucose $[\mathrm{mg} / \mathrm{dl}]$ : } \\
\hline$\leq 99$ & $23(35.4 \%)$ & $51(79.69 \%)$ & \\
\hline Above normal range (> 99 mg/dl) & $42(64.6 \%)$ & $13(20.31 \%)$ & $<0.0001$ \\
\hline \multicolumn{4}{|l|}{$\mathrm{OR}=7.16 ; 95 \% \mathrm{Cl}: 3.24-15.84$} \\
\hline \multicolumn{4}{|l|}{ Protein in CSF [g/l]: } \\
\hline$\leq 0.8 \mathrm{~g} / \mathrm{l}$ & $9(13.85 \%)$ & $48(75 \%)$ & $<0.0001$ \\
\hline Above normal range $(>0.8 \mathrm{~g} / \mathrm{l})$ & $56(86.15 \%)$ & $16(25 \%)$ & \\
\hline \multicolumn{4}{|l|}{$\mathrm{OR}=18.67 ; 95 \% \mathrm{Cl}: 7.56-46.06$} \\
\hline \multicolumn{4}{|l|}{ WBC in CSF/ $\mu \mathrm{l}:$} \\
\hline$\leq 1000$ & $5(7.69 \%)$ & $22(34.38 \%)$ & $<0.0002$ \\
\hline Above normal range $[>1000 / \mu l]$ & $60(92.31 \%)$ & $42(63 \%)$ & \\
\hline \multicolumn{4}{|l|}{$\mathrm{OR}=6.28 ; 95 \% \mathrm{Cl}: 2.20-17.93$} \\
\hline \multicolumn{4}{|l|}{ Glucose in CSF [mg/dl]: } \\
\hline$\leq 50 \mathrm{mg} / \mathrm{dl}$ & $50(76.92 \%)$ & $20(25.31 \%)$ & $<0.0001$ \\
\hline$>50 \mathrm{mg} / \mathrm{dl}$ & $15(23.08 \%)$ & $44(68.75 \%)$ & \\
\hline $\mathrm{OR}=7.33 ; 95 \% \mathrm{Cl}: 3.35-16.04$ & & & \\
\hline
\end{tabular}

CRP-C-reactive protein, CSF-cerebrospinal fluid, CI - confidence interval, OR-odds ratio, WBC - white blood cells.

\section{Discussion}

In our study a normal value of the CRP concentration appeared to be the most useful inflammation parameter to exclude bacterial meningitis (high negative predictive value). But a normal value of CRP on admission to hospital cannot exclude aseptic meningitis. C-reactive protein is one of the acute phase reactants produced by hepatocytes in response to inflammation. Its production is stimulated by IL- 1 and IL- 6 released from macrophages after activation by tissue damage, infection (bac- terial, viral, fungal) or inflammation. An increase in CRP concentration in response to generalized inflammation occurs gradually during the first $12 \mathrm{~h}$, reaching a maximum level at 48 to $72 \mathrm{~h}$ [12].

Nigrovic et al. reported that CRP concentration higher than $100 \mathrm{mg} / \mathrm{l}$ is a sensitive marker for bacterial meningitis but lacks specificity [11]. Oostenbrink et al. proposed a clinical score in which one of the criteria suggesting bacterial infection was a CRP level higher than $50 \mathrm{mg} / \mathrm{l}$ [13]. In our study all the 65 children with bacterial meningitis had CRP concentrations higher than $80 \mathrm{mg} / \mathrm{l}$, while 
Table III. Sensitivity and specificity of calculated values of selected biochemical and hematological parameters in differentiation between bacterial and aseptic meningitis

\begin{tabular}{|c|c|c|c|c|c|c|}
\hline Parameter & Sensitivity (\%) & $95 \% \mathrm{Cl}$ & Specificity (\%) & $95 \% \mathrm{Cl}$ & $+\mathrm{LR}$ & $-\mathrm{LR}$ \\
\hline $\mathrm{CRP}>84 \mathrm{mg} / \mathrm{l}$ & 98.46 & $91.7-99.7$ & 100 & $94.3-100$ & & 0.02 \\
\hline D-dimers $>970 \mu \mathrm{g} / \mathrm{l}$ & 90.77 & $81.0-96.5$ & 89.19 & $74.6-96.9$ & 8.40 & 0.10 \\
\hline Fibrinogen $>4.4 \mathrm{~g} / \mathrm{l}$ & 95.38 & $87.1-99.0$ & 80.43 & $66.1-90.6$ & 4.88 & 0.06 \\
\hline Glucose $>95$ mg/dl & 64.62 & $51.8-76.1$ & 79.69 & $67.8-88.7$ & 3.18 & 0.44 \\
\hline$W B C>13 / \mu l$ & 71.43 & $58.7-82.1$ & 82.81 & $71.3-91.1$ & 4.16 & 0.35 \\
\hline Glucose CSF $\leq 40 \mathrm{mg} / \mathrm{dl}$ & 66.15 & $53.4-77.4$ & 93.75 & $84.7-98.2$ & 10.58 & 0.36 \\
\hline Leukocytes CSF > 992 & 76.92 & $64.8-86.5$ & 90.62 & $80.7-96.5$ & 8.21 & 0.25 \\
\hline Protein CSF > $0.96 \mathrm{~g} / \mathrm{l}$ & 83.08 & $71.7-91.2$ & 90.62 & $80.7-96.5$ & 8.86 & 0.19 \\
\hline Age $\leq 74$ months & 64.62 & $51.8-76.1$ & 79.69 & $67.8-88.7$ & 3.18 & 0.44 \\
\hline BMS $>1$ & 93.85 & $85.0-98.3$ & 93.75 & $84.7-98.2$ & 15.02 & 0.07 \\
\hline
\end{tabular}

The first percentage for each marker corresponds to the marker's sensitivity. Values are the $95 \% \mathrm{Cls}$ for sensitivity. The second percentage for each marker corresponds to the marker's specificity. Values are the $95 \% \mathrm{Cl}$ for specificity. BMS - Bacterial Meningitis Score, $C R P-C$-reactive protein, CSF-cerebrospinal fluid, $C l$-confidence intrerval, $+L R$ - positive likelihood ratio, $-L R$ - negative likelihood ratio, $W B C-$ white blood cells.

$76 \%$ of children with aseptic meningitis had CRP levels within the normal range.

Data obtained in this study indicated that children with meningitis with a CRP level lower than $80 \mathrm{mg} / \mathrm{l}$ are at low risk of having bacterial etiology. Such information obtained before lumbar puncture is helpful in planning further treatment and diagnostic tests.

A high negative predictive value of CRP concentration, observed in our study, is of great value for a population with low rates of vaccination against S. pneumoniae and $N$. meningitidis, because it helps to identify severe bacterial infections at the emergency room level. Use of a cutoff CRP level in our study clearly indicated patients with bacterial meningitis, before lumbar puncture was performed.

Children with meningitis during the course of varicella were excluded, because in those children elevation of CRP can be caused by skin infection.

Procalcitonin is a 116-amino-acid peptide, calcitonin prohormone. It is more specific to bacterial infection. The increase in the procalcitonin level is very high and quick in response to bacterial endotoxins, but the increase is only minimal in patients with viral infections. Procalcitonin concentration increases rapidly in response to bacterial infection and reaches peak values within 12 to $24 \mathrm{~h}$. There are some data indicating the usefulness of procalcitonin in diagnosis of bacterial meningitis [12]. Dubos et al. found that a serum procalcitonin greater than $0.5 \mathrm{ng} / \mathrm{ml}$ predicts bacterial rather than aseptic meningitis in children [14]. Based on a study performed on 59 children with meningitis, Gendrel et al. concluded that measurement of plasma procalcitonin might be of value in the differential diagnosis of bacterial or viral meningitis [15].
The cost of the CRP test is around 10 PLN (Polish zloty), while that of the procalcitonin test is 90 PLN. So far, because of high costs, this biomarker is not used routinely at admission, so it cannot replace the widely used CRP.

Bacterial meningitis is observed mainly in children younger than 5 years old [16]. In our study the group mean age was 5 years old, which may be due to the fact that the lower limit was 28 days. Newborns with meningitis are hospitalized at neonatal units, whereas our study was based at an infectious diseases department. Older age of children with aseptic meningitis can be explained by the mechanism of such infections. Aseptic meningitis is a result of a strong immunologic response against the causative agent. It is not observed in people in immunosuppression or in young children with an immature immunologic system [17].

The Bacterial Meningitis Score was validated in a population with high rates of vaccination against the main bacterial pathogens: S. pneumoniae, $N$. meningitidis and $H$. influenzae [18]. In Poland only vaccinations against $H$. influenzae are compulsory, whereas streptococcal and meningococcal vaccinations are available but are expensive, and thus vaccinations rates are low. That is why the rates of bacterial meningitis in Polish children are higher compared with other developed countries [19].

The incidence of bacterial meningitis in Poland in children younger than 5 years old is estimated at $9 / 100000$ population, which is higher than in the USA $(1.38 / 100000)[4,7,9,18]$.

Among analyzed children with bacterial meningitis none had less than one point in the BMS, and the mean value was 2.97. Among children with aseptic meningitis only 22 (34\%) had at least 
one point in the BMS. The BMS has application in children with overall good condition, who can be treated in an outpatient setting. The exclusion criteria are hemorrhagic rash and coexisting diseases requiring hospitalization. With these exclusions $84 \%$ of children with aseptic meningitis did not score any points.

Our study proved high sensitivity and specificity of the rule derived by Nigrovic also in a population with low rates of vaccination against S. pneumoniae and N. meningitidis.

Bonsu et al. developed a prediction score based on common cerebrospinal fluid and peripheral blood and intended for children with unavailable, negative cerebrospinal fluid Gram stain results. The rule was validated in 158 children. The values were highly discriminant in all cases [16].

The observed clinical symptoms are not useful in distinguishing bacterial from aseptic meningitis. Headache and vomiting were more common in aseptic meningitis, but this may be age-dependent. Aseptic meningitis is more common among older children, and they can complain of a headache.

Hemorrhagic rash was more common in children with bacterial meningitis ( $40 \%$ vs. $6 \%$ ), but it was present at admission only in a minority of cases. Hence absence of rash at admission to hospital is not sufficient to exclude bacterial etiology. Occurrence of seizures depends more on patients' age than on meningitis etiology.

Cerebrospinal fluid culture remains the gold standard in diagnosis of meningitis, but bacterial cultures are time consuming. This test is an invasive one and sometimes gives false negative results, especially after antibiotic administration. Fast latex kits or polymerase chain reaction (PCR) testing of cerebrospinal fluid are more sensitive than culture, particularly after antibiotic administration [20].

With the increasing percentage of unvaccinated children, bacterial meningitis may still remain a problem [21]. Clinicians must be aware that in a large group of children presenting at the emergency department with symptoms of neuroinfection, there will be a few with severe, potentially life-threatening disease. Therefore tools to identify them as soon as possible are urgently needed. Thus application of a prediction model consisting of BMS and CRP level could theoretically prevent unnecessary antibiotic treatment in aseptic meningitis, without a risk of leaving children with bacterial meningitis without treatment. The diagnostic usefulness of the studied parameters is limited to a population with a low rate of vaccination coverage against $S$. pneumoniae and $N$. meningitidis. A larger, multi-center study performed on different populations of children is needed to further validate this clinical decision rule.

In conclusion, in children with meningitis, inflammatory biomarkers such as CRP, fibrinogen and D-dimers differ statistically significantly depending of the etiology - bacterial or aseptic. Normal values give strong arguments to exclude a bacterial origin. Treatment with antibiotics will not be useful. Serum concentration of CRP higher than $80 \mathrm{mg} / \mathrm{l}$ is a useful marker of bacterial etiology of meningitis. Positive CRP offers sufficient arguments to start antibiotic treatment. A high BMS is indicative of a bacterial meningitis. A prediction model consisting of BMS and CRP level can be useful in discriminating between bacterial and aseptic meningitis in hospitalized children.

\section{Conflict of interest}

The authors declare no conflict of interest.

\section{References}

1. Sáez-Llorens $X, M c C r a c k e n ~ G H$. Bacterial meningitis in children. Lancet 2003; 361: 2139-48.

2. Mook-Kanamori BB, Geldhoff M, van der Poll T, van de Beek D. Pathogenesis and pathophysiology of pneumococcal meningitis. Clin Microbiol Rev 2011; 24: 557-91.

3. Stephens DS, Greenwood B, Brandtzaeg P. Epidemic meningitis, meningococcaemia, and Neisseria meningitides. Lancet 2007; 369: 2196-210.

4. Grzesiowski P. Invasive pneumococcal disease in children up to 5 years in Poland. Eur J Clin Microb Infect Dis 2008; 27: 883-5.

5. Negrini B, Kelleher KJ, Wald ER. Cerebrospinal fluid findings in aseptic versus bacterial meningitis. Pediatrics 2000; 105: 316-9.

6. Curtis S, Stobart K, Vandermeer B, Simel DL, Klassen T. Clinical features suggestive of meningitis in children: a systematic review of prospective data. Pediatrics 2010; 126: 952-60.

7. McIntyre PB, O’Brien KL, Greenwood B, van de Beek D. Effect of vaccines on bacterial meningitis worldwide. Lancet 2012; 380: 1703-11.

8. Peltola H, Salo E, Saxen H. Incidence of Haemophilus influenzae type $b$ meningitis during 18 years of vaccine use: observational study using routine hospital data. BMJ 2005; 330: 18-9.

9. Hsu HE, Shutt KA, Moore MR, et al. Effect of pneumococcal conjugate vaccine on pneumococcal meningitis. N Engl J Med 2009; 360: 244-56.

10. Pochling KA, Talbot TR, Griffin MR, et al. Invasive pneumococcal disease among infants before and after introduction of pneumococcal conjugate vaccine. JAMA 2006; 295: 1668-74.

11. Nigrovic LE, Kuppermann N, Macias CG, et al. Clinical prediction rule for identifying children with cerebrospinal fluid pleocytosis at very low risk of bacterial meningitis. JAMA 2007; 297: 52-60.

12. Shimetani N, Shimetani K, Mori M. Levels of three inflammation markers, C-reactive protein, serum amyloid A protein and procalcitonin, in the serum and cerebrospinal fluid of patients with meningitis. Scand J Clin Lab Invest 2001; 61: 567-74.

13. Oostenbrink R, Moons KG, Derksen-Lubsen AG, Grobbee $\mathrm{DE}$, Moll HA. A diagnostic decision rule for management of children with meningeal signs. Eur J Epidemiol 2004; 19: 109-16. 
14. Dubos F, Korczowski B, Aygun DA, et al. Serum procalcitonin level and other biological markers to distinguish between bacterial and aseptic meningitis in children: a European multicenter case cohort study. Arch Pediatr Adolesc Med 2008; 162: 1157-63.

15. Gendrel D, Raymond J, Assicot M, et al. Measurement of procalcitonin levels in children with bacterial or viral meningitis. Clin Infect Dis 1997; 24: 1240-2.

16. Bonsu BK, Harper MB. Differentiating acute bacterial meningitis from acute viral meningitis among children with cerebrospinal fluid pleocytosis: a multivariable regression model. Pediatr Infect Dis J 2004; 23: 511-7.

17. Centers for Disease Control and Prevention. Outbreaks of aseptic meningitis associated with echoviruses 9 and 30 and preliminary surveillance reports on enterovirus activity - United States, 2003. MMWR Morb Mortal Wkly Rep 2003; 52: 761-4.

18. Meldunki epidemiologiczne. Lata 2007-2011. Narodowy Instytut Zdrowia Publicznego. PZH Warszawa. www.pzh. gov.pl/oldpage/epimeld/index_p.html, 2011-08-09.

19. Dubos F, Lamotte B, Bibi-Triki F, et al. Clinical decision rules to distinguish between bacterial and aseptic meningitis. Arch Dis Child 2006; 91: 647-50.

20. Poppert S, Essig A, Stoehr B, et al. Rapid diagnosis of bacterial meningitis by real-time PCR and fluorescence in situ hybridization. J Clin Microbiol 2005; 43: 3390-7.

21. Brouwer MC, Thwaites GE, Tunkel AR, van de Beek D. Dilemmas in the diagnosis of acute community-acquired bacterial meningitis. Lancet 2012; 380: 1684-92. 